\title{
Multiwavelength observations of the microquasar GX 339-4 during its recent 2010 outburst
}

\author{
M. Cadolle Bel* \\ ESAC/ISOC, Spain \\ E-mail: Marion.Cadolle@sciops.esa.int
}

\section{J. Rodriguez and S. Corbel}

CEA-Saclay \& AIM, France

\section{P. d'Avanzo}

INAF, Italy

\section{J. Tomsick}

SSL/Univ. California, USA

\section{M. Russell}

Univ. of Amsterdam, The Netherlands

\section{F. Lewis}

Faulkes Telescope Project, Univ. of Glamorgan, Wales

\section{E. Kuulkers}

\section{ESAC/ISOC, Spain}

In the light of recent observations obtained with the space observatories INTEGRAL, RXTE and Swift, as well as ground-based instruments operating in radio and NIR/Optical, we present our progress made in the understanding of the extreme gravity phenomena in GX 339-4 in distinct spectral states. We show a preliminary analysis of the extensive monitoring obtained as part of the 2010 Galactic Bulge monitoring Program, the Galactic Halo Scan Key Program and two Targets of Opportunity (ToOs) we triggered with INTEGRAL. These latter were simultaneously covered by other observatories operating in X-rays, down to the radio domain. GX 339-4 underwent its bright outburst starting in February 2010 and transited from the Hard to the Soft State in April. We show the evolution of the spectral parameters and changes in the X-ray binary system components (jet, disc, hot medium). This is crucial for our understanding of the physics of the hot plasma (jet base and/or corona). Light curves, spectra and time studies are shown and discussed in the context of high-energy phenomena, and future perspectives of probing accretion/ejection processes around microquasars.

8th INTEGRAL Workshop, The Restless Gamma-ray Universe, September 27-30, 2010

Dublin Ireland

\footnotetext{
*Speaker.
} 

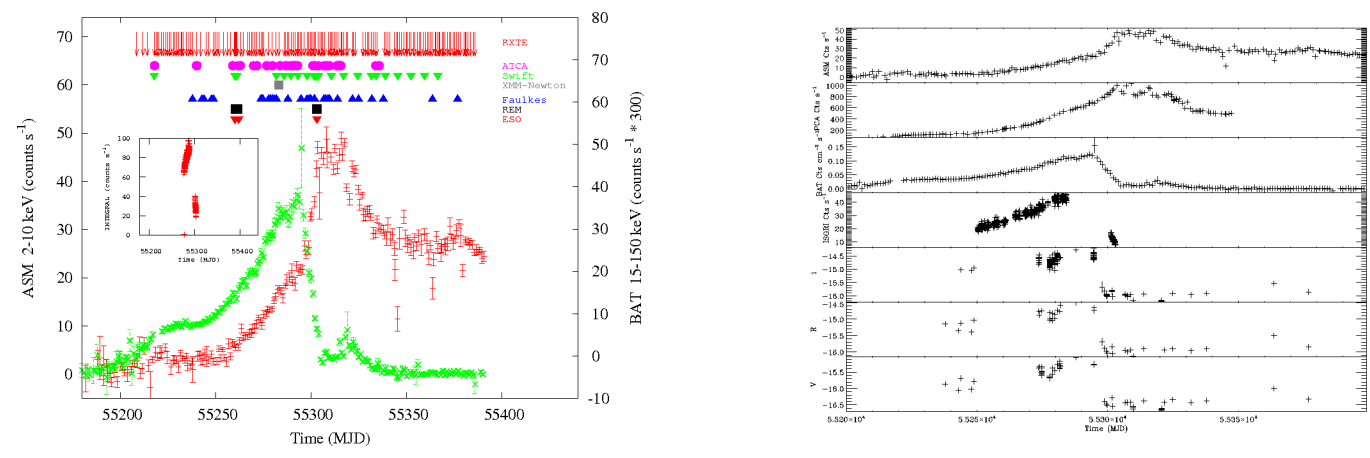

Figure 1: Left: RXTE/

ASM (red), Swift/BAT (green) and INTEGRAL/ISGRI (red, small panel) light curves of GX 339-4 in outburst. ATCA, FT South, REM and ESO/FORS2 observations are indicated. Right: Detailed ASM, PCA, BAT, ISGRI (18-40 keV) and FT South light curves.

\section{Introduction}

X-ray Transients (XTs) are accreting Low-Mass X-ray Binaries (LMXB) that spend most of their time in a faint, quiescent state. They undergo large amplitude outbursts with rise times of only a few days or weeks, with typical recurrence periods of many years (Tanaka \& Shibazaki 1996). Depending on the relative strengths of each component (disc, hot "corona" and/or base of the jet) and on how they vary, several spectral states have been identified (see, e.g., McClintock \& Remillard (2006), Homan \& Belloni (2005)): the Low/Hard state (LHS), dominated by nonthermal emission and the High Soft State (HSS), dominated by emission from the accretion disc. In the LHS, the fast time variability is dominated by strong ( $\sim 30 \%$ fractional $\mathrm{rms}$ ) band-limited noise. At times, low-frequency quasi-periodic oscillations are present; flat-spectrum radio emission is observed associated with compact jet ejection (Corbel et al. 2000, 2003; Gallo et al. 2003, 2006; Fender et al. 2004). In the HSS, only weak power law noise is present in the power spectrum. No core radio emission is detected (see Fender et al. 1999, 2005). Further states have been identified as "intermediate" based on the above-mentioned differences in the soft/hard X-ray components, variability and radio emission: the HIMS and SIMS (Hard and Soft InterMediate States). Since the beginning of 2010, GX 339-4 entered a new outburst. We therefore triggered our ToOs for XTs in outburst with INTEGRALassociated to large multi-wavelength campaigns. It was extensively observed by INTEGRAL (Prat et al. 2010; Cadolle Bel et al. 2010) as well as by other instruments (Shaposhnikov \& Tomsick 2010; Motta et al. 2010a; Yu 2010; Belloni et al. 2010; Motta et al. 2010b) and facilities on ground (Lewis et al. 2010; Corbel et al. 2010a; Russell et al. 2010; Buxton et al. 2010). We report here the preliminary results of some observations of GX 339-4 together with $S$ wift, RXTE and NIR/optical/radio data, starting with the description of the data and analysis procedures employed (Sect. 2) followed by our results (Sect. 3) and conclusions (Sect. 4).

\section{Observation and Data Reduction}

Figure 1 (left) shows the RXTE/ASM, Swift/BAT and INTEGRAL/ISGRI light curves of the outburst, with the time of the multiwavelength observations indicated. X-ray together with FT 

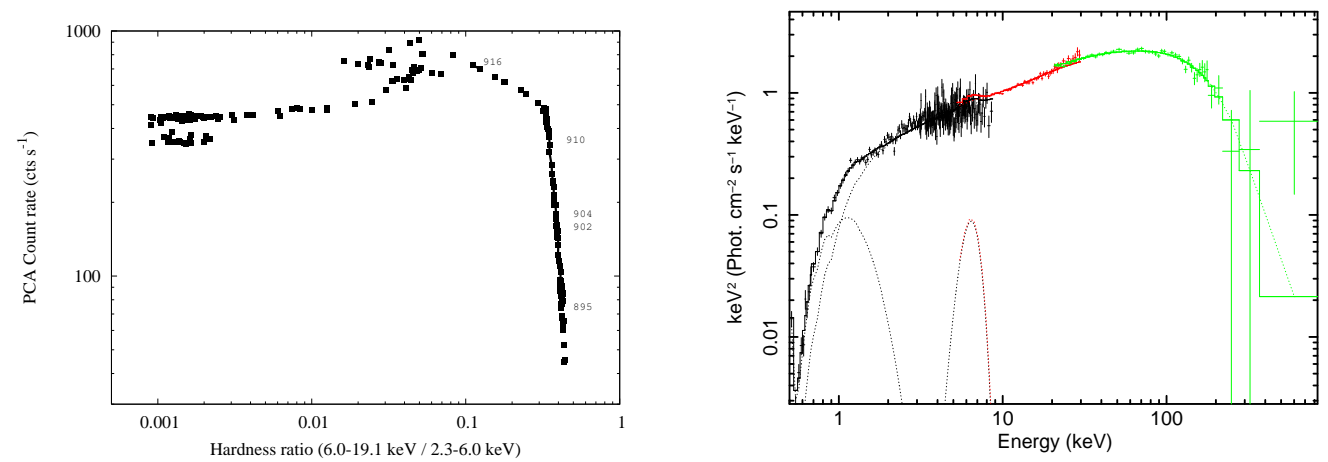

Figure 2: Left: HID of GX 339-4 on a logarithmic scale obtained with the top layer of RXTE/PCA (2.3-19.1 keV, detector 2) between 55208 and 55347 MJD (numbers=INTEGRAL revolutions). The source globally evolve from bottom to top and from right to left. Right: Swift (black), RXTE (red) and INTEGRAL (green) spectra of GX 339-4 (Rev. 902) in LHS fitted with disc, reflection, Fe line and Comptonization.

South detailed light curves are shown in Fig. 1 (right). INTEGRAL data were reduced with the standard analysis procedures of the Off-Line Scientific Analysis OSA 9.0 (algorithms described in Goldwurm et al. (2003) and Westergaard et al. (2003)). Systematic errors of $2 \%$ were added for both JEM-X (in the 5-25 keV range) and ISGRI (in the 18-400 keV range). We used the maps, the response matrices and the off-axis and background corrections from OSA 9.0. The constancy of the spectral shape during a single revolution permitted us to accumulate spectra over this long time scale, to improve the signal-to-noise ratio. We analysed all available $R X T E$ observations from MJD 55217 to 55345. They were reduced with the HEASOFT software package v6.4, following standard procedures (see Rodriguez et al. 2008). Energy spectra were only extracted from detector 2 of the PCA top layer. We used the Crab spectra to determine the level of systematic errors (level of $0.8 \%$ adopted). The resultant PCA spectra of a single observation were fitted simultaneously between 3-40 keV. The XRT (0.3-10 keV) data were processed with the xrtpipeline (v0.9.9) task applying standard calibration, filtering and screening criteria. As the data suffered from heavy pile-up, we extracted photons from an annular region with inner and outer radius of 25 and 80 pixels respectively. Optical and NIR observations were performed with the REM, FT South and UVO telescopes. Image reduction was carried out by following the standard procedures. Aperture photometry was made with the SExtractor package (Bertin \& Arnouts (1996)) for all the objects in the field. The calibration was done against Landolt standard stars for the optical filters and against the 2MASS catalog for NIR filters.

\section{Results and Discussion}

Figure 1 shows that the source displayed the typical features of a XT: a strong flux increase in hard, then soft X-rays, simultaneously to high values of magnitudes (with some variabilities) that dropped after the transition. Unabsorbed bolometric fluxes evolved from 0.7 to $2.8 \times 10^{-8} \mathrm{erg}$ $\mathrm{cm}^{-2} \mathrm{~s}^{-1}$. To get an idea of the spectral behavior of the source, we produced a Hardness-Intensity Diagram (HID) with RXTE/PCA (Fig. 2, left) similar to those widely used in the literature (Fender et al. 2004). While there was no coverage from the start of the outburst to the peak, GX 339-4 did follow the usual path of XTs in outburst on this diagram (see, e.g., Belloni et al. 2005b). 
We analyzed and fitted all the available spectra with XSPEC V11.3.2 (Arnaud 1996) from MJD 55217 to 55345. The absorption found by XRT was varying between 3.7 and $9.5 \times 10^{21} \mathrm{~cm}^{-2}$, compatible to the common value used in the literature (Zdziarski et al. 1998), which is however still debated. The best modeling depended on the position of the source in the HID as the spectral parameters evolved with time: an example of spectra is shown in Fig. 2 (Right) where the best-fit was a multicolor black body disc (Mitsuda et al. 1984) plus an iron line (simple Gaussian model) and Comptonization (Titarchuk 1994) convolved by reflection (Magdziarz \& Zdziarski 1995). Throughout the outburst, a regular increase of the disc temperature from $\sim 0.2$ to $1.1 \mathrm{keV}$ was observed; it then decreased. This increase corresponds to what is usually observed for GX 339-4 in outburst: first in the HIMS, the source transits to softer states where the disc dominates the emission, and then gradually cools down before returning to quiescence. At the same time, at higher energies, the power law photon index varied between 1.4 and 2.4, thereby confirming the source transited from hard to softer states (in fact from HIMS to SIMS, HSS, SIMS and back to HIMS; Cadolle Bel et al. 2011 in prep.); this is confirmed by the evolution of the Comptonization parameters. XTs tend to evolve towards softer -but sometimes harder states again- in the late stages of their outbursts, before they return to quiescence: indeed, in their decaying phase, XTE J1720-318, XTE J1650-500 and SWIFT J1753.5-0127 showed a slowly receding disc with a decreasing inner temperature, while at the same time the relative amount of the power law contribution increased. In March, the source showed the brightest level of radio emission (Corbel et al. 2010b) ever found so far in its LHS (Corbel et al. 2003). Later in June, no radio emission was detected (Corbel et al. 2010b), consistent with the source being in the HSS. The rapid drop in optical flux and color change at the start of the state transition is reminiscent of previous outbursts (Coriat et al. 2009) and is an indication that the OIR-emitting synchrotron jet is fading (Russell et al. 2010). Indeed, the recent appearance of this radio source close to the BH suggests that we witnessed the impact of material ejected by the system with a denser phase of the interstellar medium, such as previously observed in XTE J1550-564 (Corbel et al. 2002)).

We included all the simultaneous (de-reddened) data we gathered to build the Spectral Energy Distributions (hereafter SED), with the radio, Opt./NIR, X-ray and $\gamma$-ray data. At the time of this paper, we could not include the ESO data, but first analysis are consistent with REM and FT South ones (Rahoui, private communication). We produced several SEDs at distinct epochs, some of which are shown in Fig. 3; we fitted them with broken power laws. Of course, we observed important changes in the disc and hot medium components. When the jet was present, the fits (alpha $=-0.52 \pm 0.33$ ) corresponded more to an optically thin emission. Fig. 3 (Left) is remarkably similar to the Fig. 2 of Corbel \& Fender (2002), focusing on GX 339-4 jet signatures; from Fig. 3 (Right), the jet was then fading. The other component (probably the disc) stayed about the same. The $H$-band faded by a factor of 10 whereas the $V$-band was only fading by a factor of $\sim$ 2 at most. We saw the jet contribution move to lower frequencies: the earliest data still have the jet dominating in $H$-band in NIR, but not in Optical anymore. The shape of the SEDs in LHS is similar to the one of the transient LMXB XTE J1118+480 (Chaty et al. 2003; Zurita et al. 2006) in terms of component evolutions. Hynes et al. (2002) observed XTE J1859+226 during the decline from its 1999-2000 outburst: in the soft state, the slopes derived were always between 0.1 and 0.5 , which is slightly different from our value (closer to the peak of SIMS). 

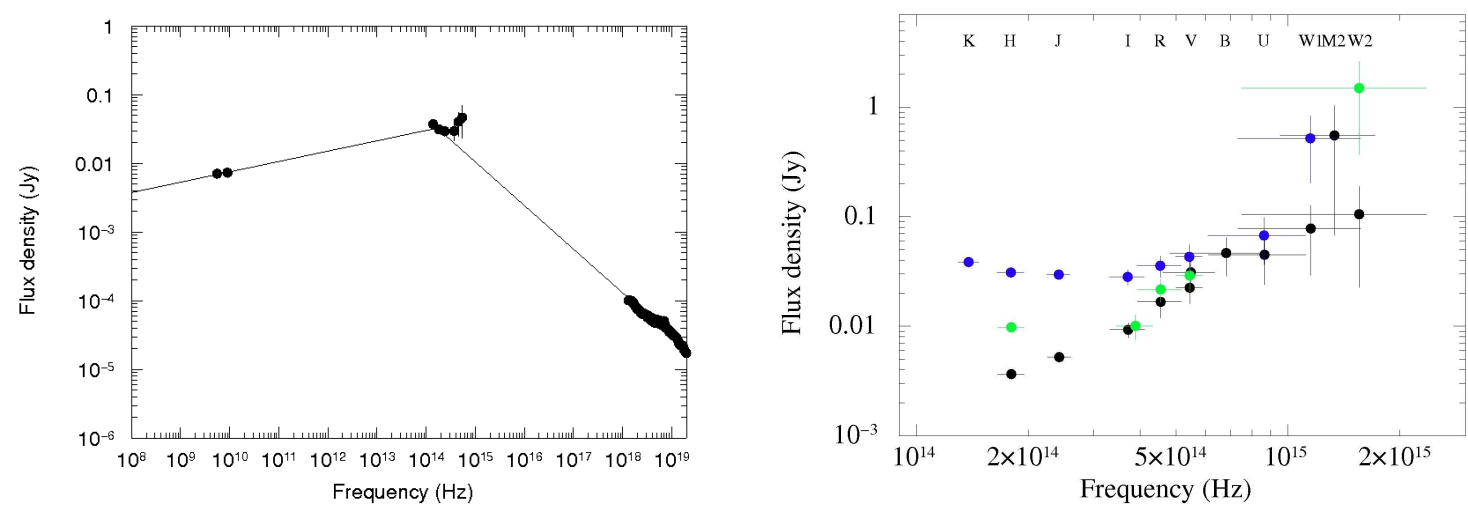

Figure 3: Left: ATCA (Corbel, private communication), REM and RXTE data fitted with broken power laws (index1 $=0.15$, index2 $=-0.64$ ) around MJD 55259.9-55261.1. Right: Compiled SEDs at MJD=55260.21, 55397.96 (green) and 55303.30 (UVOT, REM and FT South data only).

\section{Conclusions}

During its 2010 outburst, GX 339-4 showed typical light curves, HID and spectral evolution of a LMXB in outburst, transiting between LHS, SIMS, HSS then back to HIMS. Preliminary SEDs fitting shows evolutions for each component, with the presence then disappearance of the jet. The diversity and complexity of the interplay of the different emitting media during state transitions need more campaigns and observations to be better understood. We thank the INTEGRAL, Swift and RXTE mission planners for programming the ToO observations described in the paper. The present work is based on observations with INTEGRAL, an ESA project with instruments and science data center funded by ESA member states (especially the PI countries: Denmark, France, Germany, Italy, Switzerland, Spain, Czech Republic and Poland, and with the participation of Russia and the USA), and with RXTE. This research has made use of the NASA Astrophysics Data System Abstract Service and of the SIMBAD database operated at the CDS, Strasbourg, France.

\section{References}

Arnaud, K. A. 1996, ASP Conferences, 101, 17

Belloni, T., Homan, J., Casella, P., et al. 2005b, A\&A, 440, 207

Belloni, T., Motta, S., \& Muñoz-Darias, T. 2010, The Astronomer's Telegram, 2577, 1

Bertin, E. \& Arnouts, S. 1996, , 117, 393

Buxton, M., Dincer, T., Kalemci, E., \& Tomsick, J. 2010, The Astronomer's Telegram, 2549, 1

Cadolle Bel, M., Kuulkers, E., Ibarra, A., et al. 2010, The Astronomer's Telegram, 2573, 1

Chaty, S., Haswell, C. A., Malzac, J., et al. 2003, , 346, 689

Corbel, S., Broderick, J., Brocksopp, C., Tzioumis, T., \& ., R. F. 2010a, The Astronomer's Telegram, 2525 , 1

Corbel, S., Broderick, J., Calvelo, D., et al. 2010b, The Astronomer's Telegram, 2745, 1 
Corbel, S. \& Fender, R. P. 2002, , 573, L35

Corbel, S., Fender, R. P., Tzioumis, A. K., et al. 2000, , 359, 251

Corbel, S., Fender, R. P., Tzioumis, A. K., et al. 2002, Science, 298, 196

Corbel, S., Nowak, M. A., Fender, R. P., Tzioumis, A. K., \& Markoff, S. 2003, , 400, 1007

Coriat, M., Corbel, S., Buxton, M. M., et al. 2009, , 400, 123

Fender, R., Belloni, T., \& Gallo, E. 2005, , 300, 1

Fender, R., Corbel, S., Tzioumis, T., et al. 1999, , 519, L165

Fender, R. P., Belloni, T. M., \& Gallo, E. 2004, , 355, 1105

Gallo, E., Fender, R. P., Miller-Jones, J. C. A., et al. 2006, , 370, 1351

Gallo, E., Fender, R. P., \& Pooley, G. G. 2003, , 344, 60

Goldwurm, A., David, P., Foschini, L., et al. 2003, , 411, L223

Homan, J. \& Belloni, T. 2005, Astrophysics and Space Science, 300, 107

Hynes, R. I., Haswell, C. A., Chaty, S., Shrader, C. R., \& Cui, W. 2002, MNRAS, 331, 169

Lewis, F., Russell, D. M., \& Cadolle Bel, M. 2010, The Astronomer's Telegram, 2459, 1

Magdziarz, P. \& Zdziarski, A. A. 1995, , 273, 837

McClintock, J. E. \& Remillard, R. A. 2006, Black hole binaries, Compact stellar X-ray sources. Edited by Walter Lewin \& Michiel van der Klis: Cambridge University Press, 157

Mitsuda, K., Inoue, H., Koyama, K., et al. 1984, , 36, 741

Motta, S., Belloni, T., \& Muñoz Darias, T. 2010a, The Astronomer's Telegram, 2545, 1

Motta, S., Belloni, T., Muñoz-Darias, T., \& Homan, J. 2010b, The Astronomer's Telegram, 2593, 1

Prat, L., Cadolle Bel, M., Terrier, R., et al. 2010, The Astronomer's Telegram, 2455, 1

Rodriguez, J., Shaw, S. E., Hannikainen, D. C., et al. 2008, , 675, 1449

Russell, D. M., Buxton, M., Lewis, F., \& Altamirano, D. 2010, The Astronomer's Telegram, 2547, 1

Shaposhnikov, N. \& Tomsick, J. A. 2010, The Astronomer's Telegram, 2523, 1

Tanaka, Y. \& Shibazaki, N. 1996, , 34, 607

Titarchuk, L. 1994, , 434, 570

Westergaard, N. J., Kretschmar, P., Oxborrow, C. A., et al. 2003, , 411, L257

Yu, W. 2010, The Astronomer's Telegram, 2556, 1

Zdziarski, A. A., Poutanen, J., Mikolajewska, J., et al. 1998, , 301, 435

Zurita, C., Torres, M. A. P., Steeghs, D., et al. 2006, , 644, 432 